\title{
Electron Diffraction Tomography Measurement of Defect Distribution Produced by in situ Ion Irradiation in Thin Foil.
}

\author{
Marquis Kirk*, Meimei Li**, and Pete Baldo* \\ *Materials Science Division, Argonne National Laboratory, Argonne, IL 60439 \\ ** Nuclear Engineering Division, Argonne National Laboratory, Argonne, IL 60439
}

In our recent experimental work to utilize the IVEM-Tandem Facility for comparing and benchmarking computer model simulations of in situ ion irradiated thin foils, the role of the loss of mobile defects and defect clusters to nearby foil surfaces has obvious importance. The effect of foil surfaces was previously indirectly measured in the usual plane view images of nanometer sized defect clusters as a function of varying foil thickness. A direct measurement of defect density in three dimensions is more sensitively comparable to computer simulation.

While electron tomography has been used in biological specimen examinations for several decades, only recently has it been applied in materials science to image structures in $3 \mathrm{D}$ on the nanometer scale [1]. However, most recent studies employed z contrast STEM over large tilt ranges and suppress diffraction contrast effects [2]. Here we use diffraction contrast with precise control of constant diffraction conditions over a high tilt range, and apply this technique to in situ ion irradiation of thin foils where the nm sized defects are expected to vary strongly in density with foil depth and without chemical differences to allow use of $\mathrm{z}$ contrast or EFTEM.

A $3 \mathrm{~mm}$ disk of electropolished pure Mo was irradiated at $80^{\circ} \mathrm{C}$ in situ the IVEM with $1 \mathrm{MeV} \mathrm{Kr}$ ions to a fluence of $5 \times 10^{12} \mathrm{ions} / \mathrm{cm}^{2}(0.015 \mathrm{dpa})$. This produced a convenient density of defects dominated by self interstitial clusters and dislocation loops with sizes from $1.5 \mathrm{~nm}$ (TEM visibility threshold) to about $5 \mathrm{~nm}$ (Figure 1). Defect imaging conditions were precisely controlled using $g=110$ in a foil with normal near $<111>$. Diffraction conditions were approximately $5 \mathrm{~g}$ in weak beam dark field and $3 \mathrm{~g}$ in bright field kinematic. Essential to success was a surface marker to maintain area and a digital camera to maintain precise area and background contrast (diffraction condition) throughout the recordings over the tilt range.

A critical component to the success of this work is the Gatan 925 double tilt/rotation stage, a nearly ideal sample stage for the experiment. The rotation in the sample plane allowed alignment of the $<110>$ crystallographic axis with the main tilt axis of the stage. For one full tomography tilt series we precisely adjusted the main stage tilt over a range of $\pm 40^{\circ}$. Images were taken every $2^{\circ}$ in both weak beam dark field and bright field kinematic conditions.

Image alignment and volume reconstruction was performed using EM3D software [3]. The distribution of 160 defects in depth through the foil thickness of $100 \mathrm{~nm}$ was measured with EM3D. The accuracy of $\mathrm{z}$ height determination for an individual defect is $\pm 0.4 \mathrm{~nm}$, or one volume slice. The position of the surface was estimated from the change in background noise with an accuracy of about $2 \mathrm{~nm}$, or 5 volume slices. The depth distribution for 160 defects is shown in Figure 2. 
For still display and rotation movie purposes the volume reconstructed data from EM3D was imported into Chimera software [4]. Plane and cross section views with full transparency are shown in Figure 3. Movie will be shown in the talk [5].

\section{References}

[1] P. A. Midgley and M. Weyland, Ultramicroscopy 96, 3-4 (2003) 413.

[2] K. Inoke et al, Acta Materialia 54, 11 (2006) 2957.

[3] EM3D: http://em3d.stanford.edu

[4] Chimera; http://www.cgl.ucsf.edu/chimera

[5] The important assistance of Dennis Graham and Bryan Miller in the group of Ian Robertson at UIUC is gratefully acknowledged. The submitted work was supported by the U.S. Department of Energy Office of Science under Contract No. DE-ACO2-06CH11357.

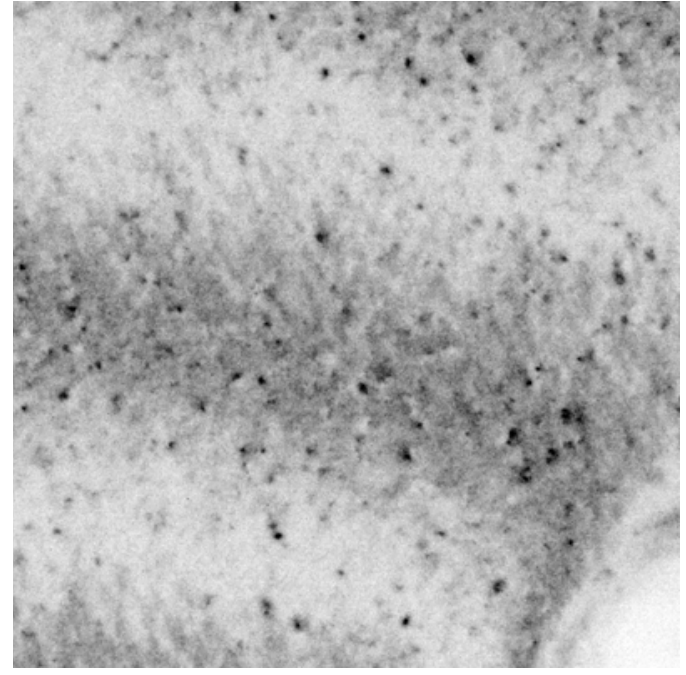

Figure 1. Inverted dark field image of defects. Image spans $340 \mathrm{~nm}$.

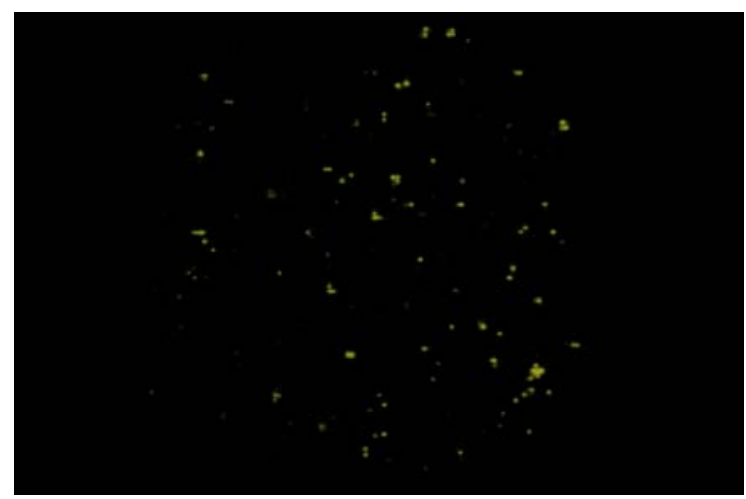

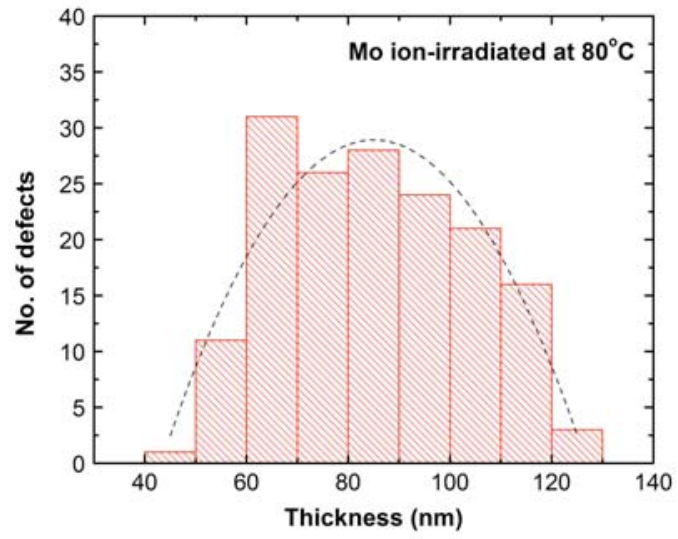

Figure 2. Distribution of defects through foil thickness. Line to guide the eye.

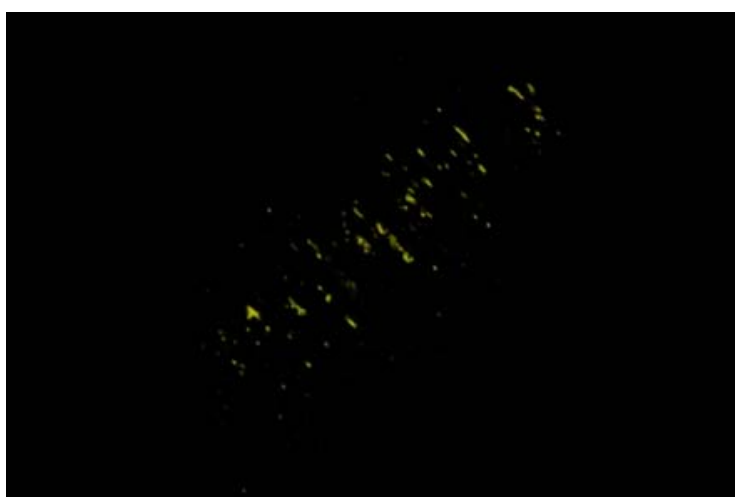

Figure 3. Plane view (left panel) and cross section view (right panel) of defects. Note some defect contrast is elongated in cross section due to reconstruction. 